\title{
Supporting Information for \\ Illuminating Excitonic Structure in Ion-Dependent Porphyrin Aggregates with Solution Phase and Single-Particle Resonance Raman Spectroscopy
}

\author{
Christopher W. Leishman and Jeanne L. McHale*
}

Department of Chemistry, Box 644630, Washington State University, Pullman, Washington 99164, United States
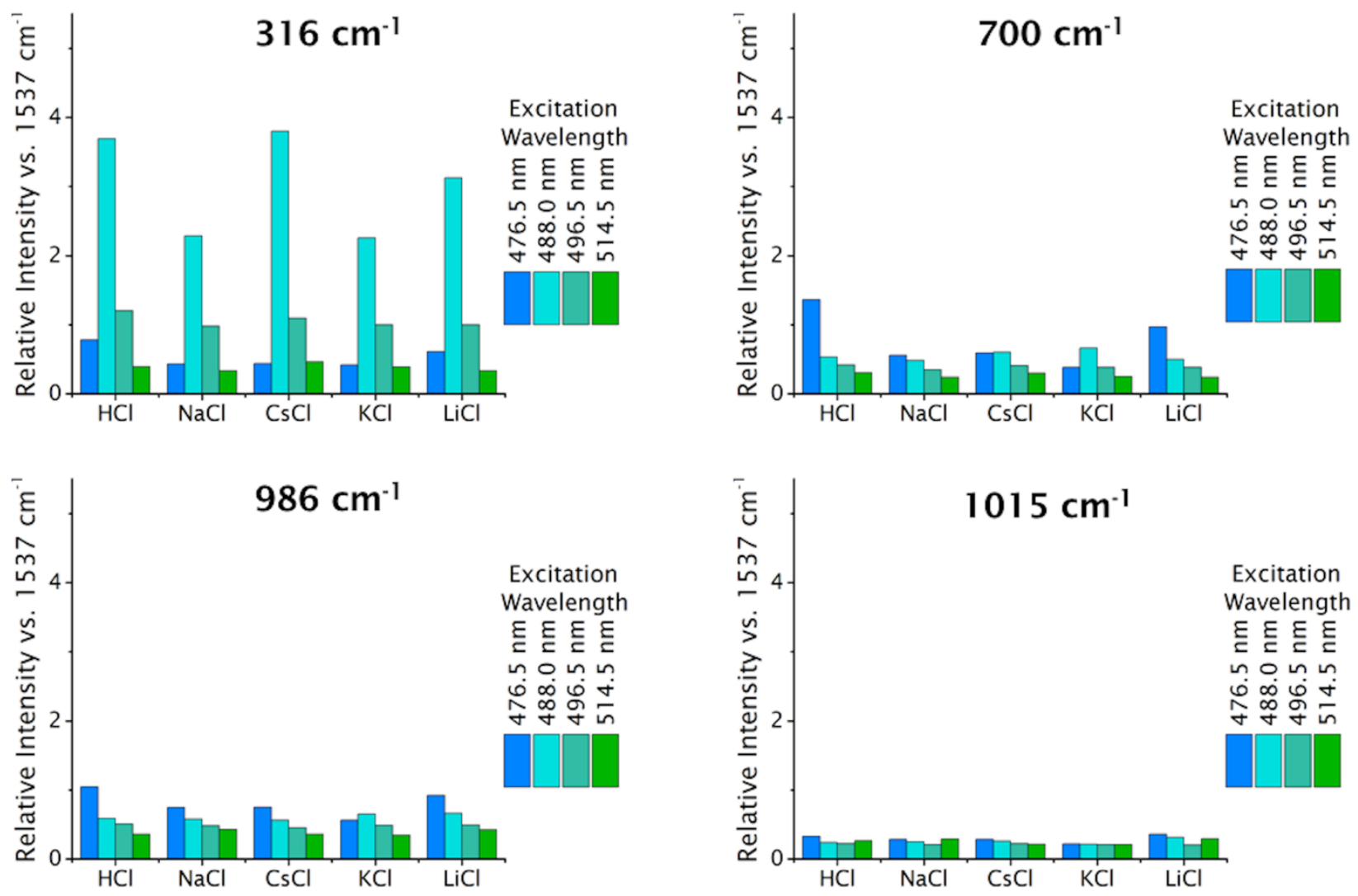

Figure S1A. Integrated relative resonance Raman intensities vs. the $\sim 1537 \mathrm{~cm}^{-1}$ mode for 316, 700, 986, and $1015 \mathrm{~cm}^{-1}$ modes from $50 \mu \mathrm{M}$ TSPP in $0.75 \mathrm{M}$ aqueous $\mathrm{HCl}, \mathrm{NaCl}, \mathrm{CsCl}, \mathrm{KCl}$, and $\mathrm{LiCl}$. 

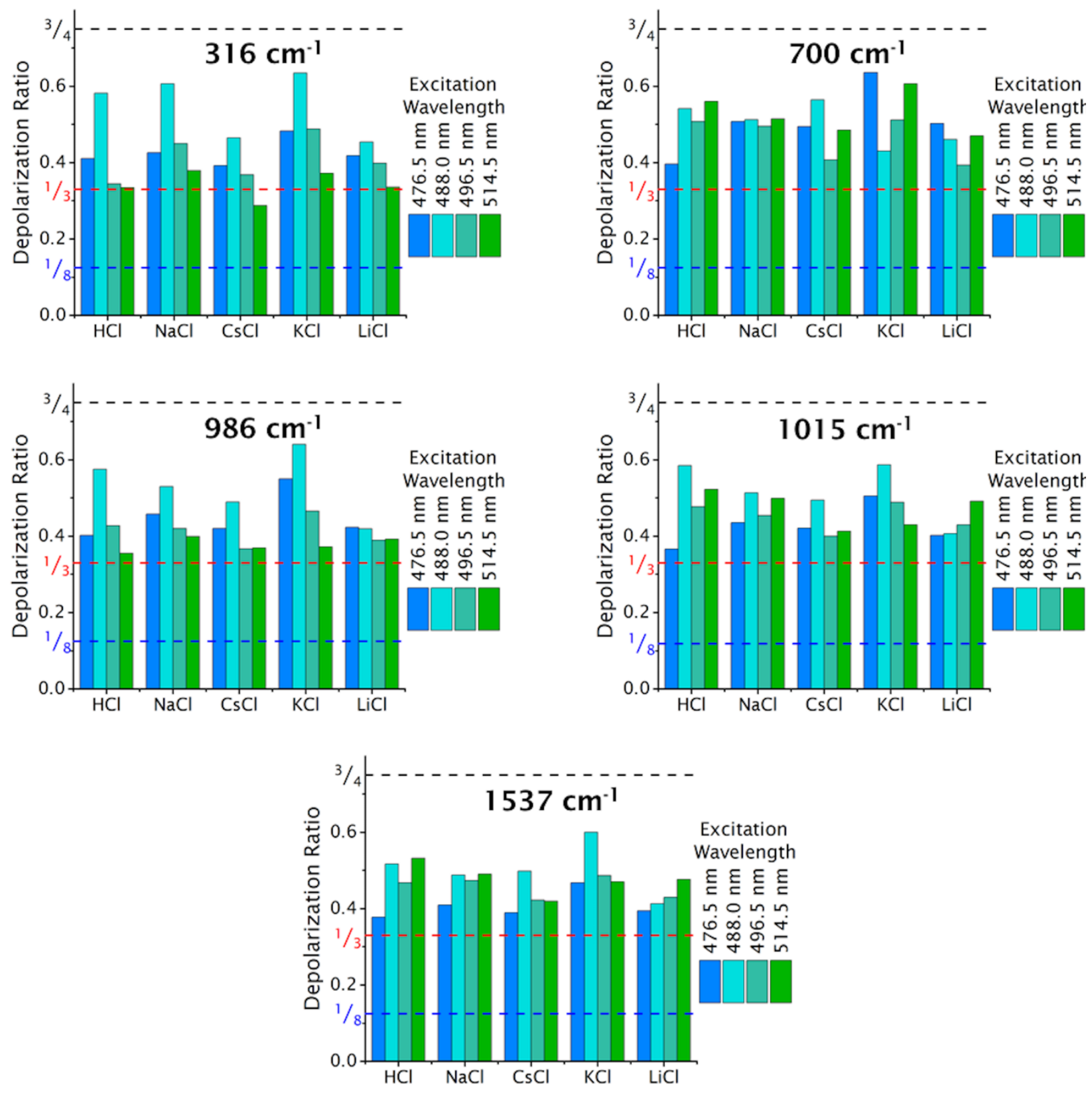

Figure S1B. Depolarization ratios vs. excitation wavelength for the 316, 700, 986, 1015, and $1537 \mathrm{~cm}^{-1}$ modes from $50 \mu \mathrm{M}$ TSPP in $0.75 \mathrm{M}$ aqueous $\mathrm{HCl}, \mathrm{NaCl}, \mathrm{CsCl}, \mathrm{KCl}$, and $\mathrm{LiCl}$.. Dashed lines indicate $\rho$ values for resonance with a non-composite electronic transition, as discussed in the text. 

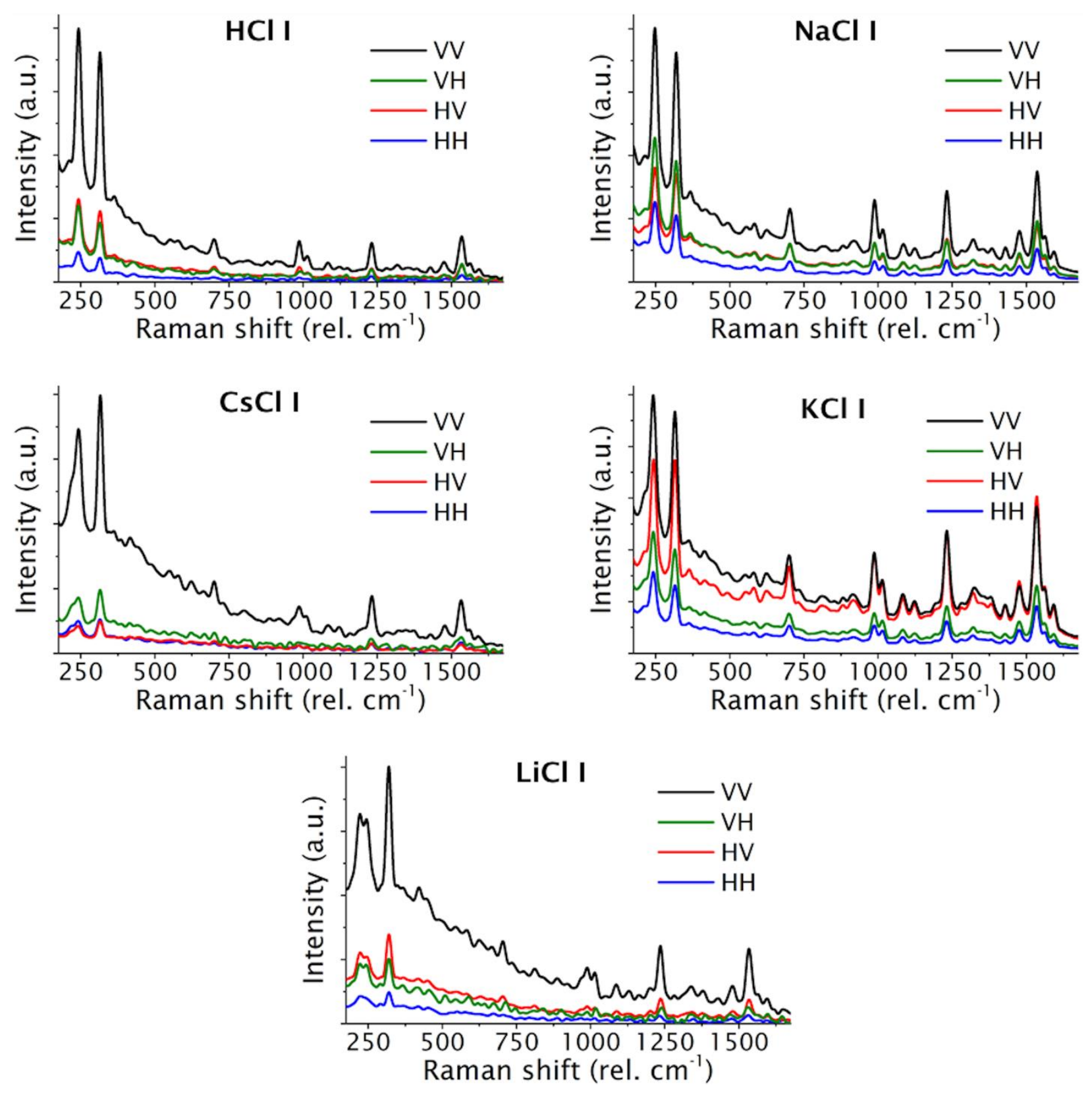

Figure S2. Full RR spectra, scaled relative to VV peak intensity, for single specimen I from each sample. 

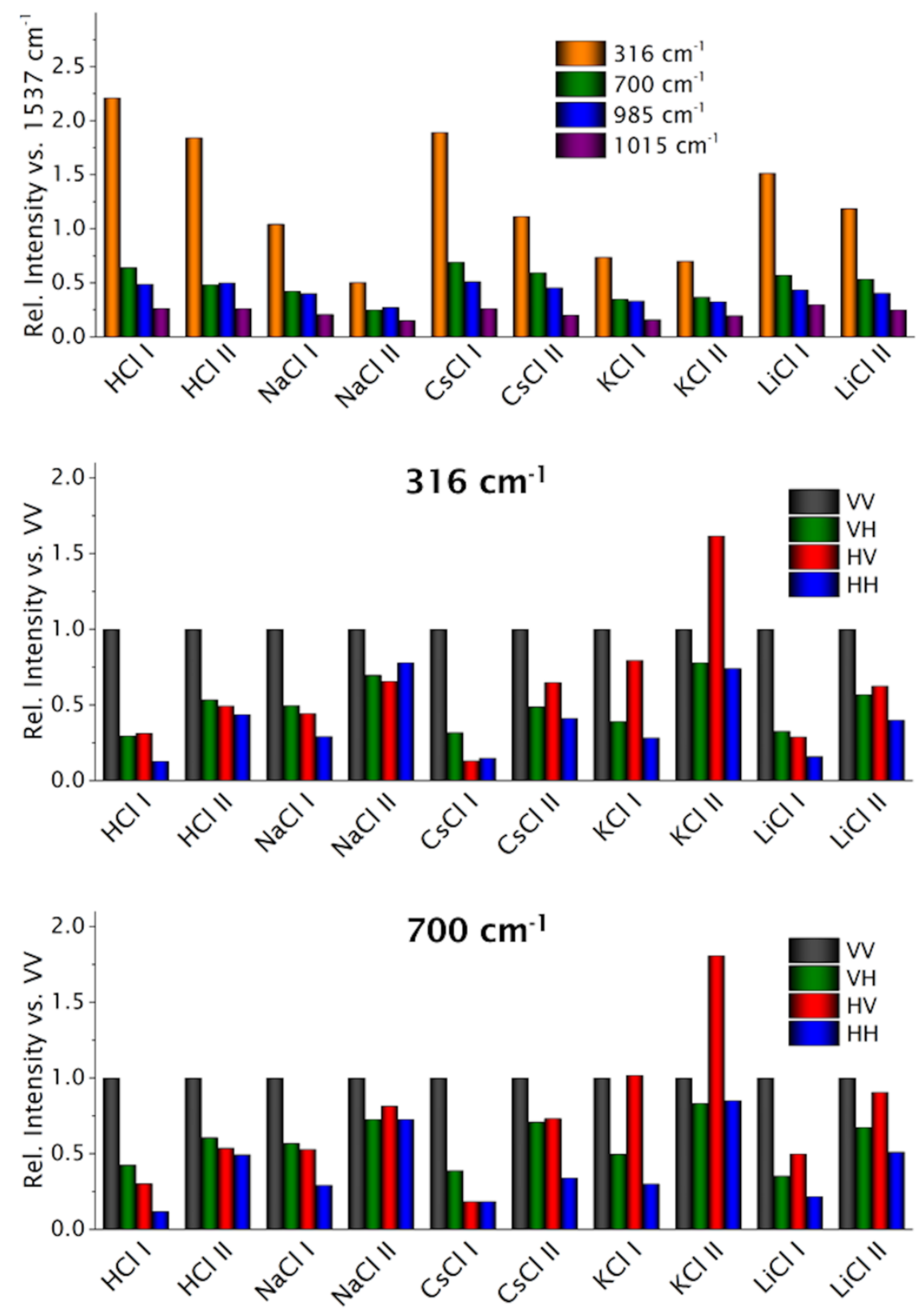

Figure S3A. Additional RR data for the ten samples shown in the images of Figure 3, for the 316, 700, 986,1015 , and $1537 \mathrm{~cm}^{-1}$ modes. Top: Relative total intensity vs. $1537 \mathrm{~cm}^{-1}$. Center and bottom: Relative RR intensities (vs. VV) corresponding to the four polarization combinations, continued on next page. 

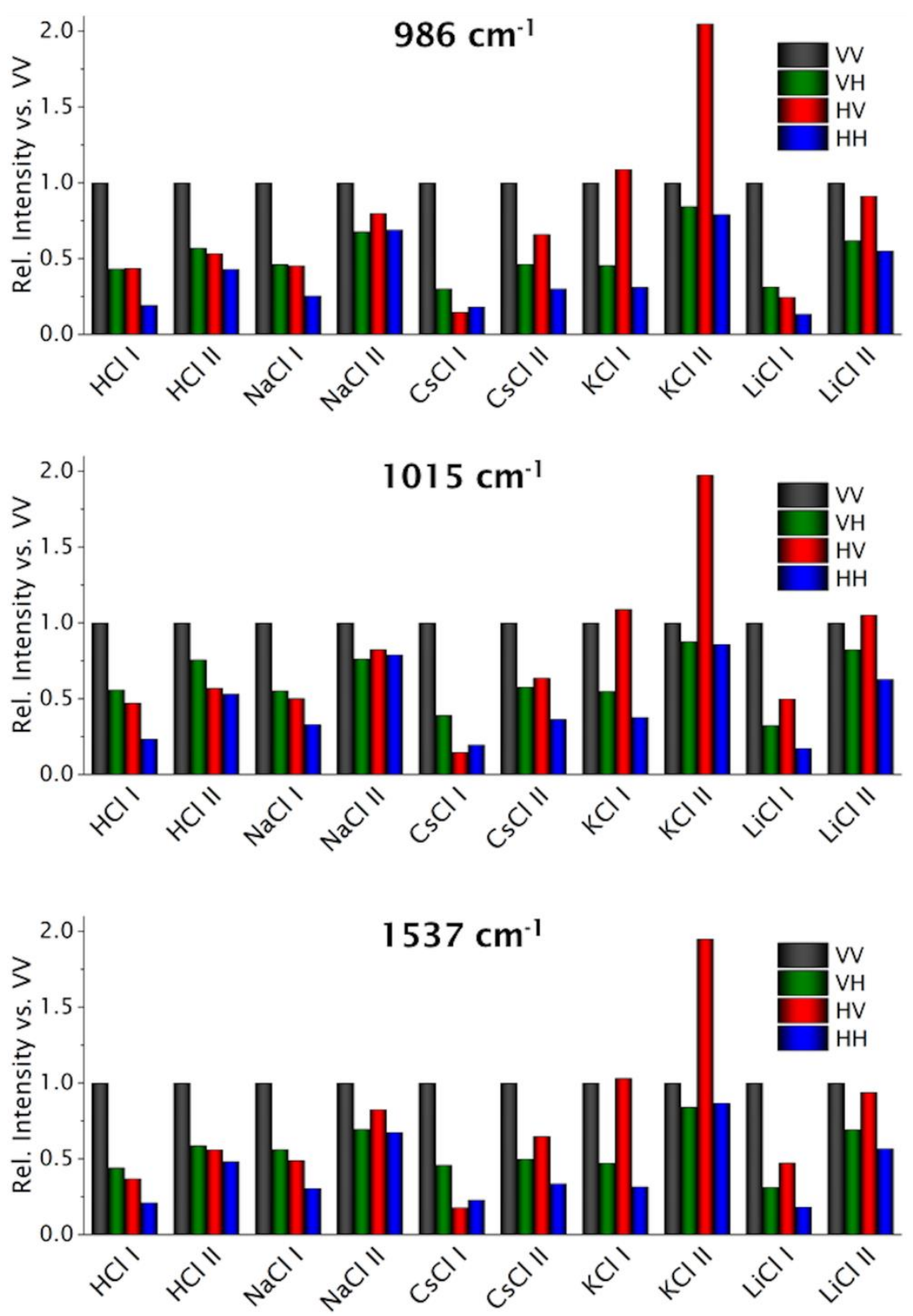

Figure S3B. Additional relative RR intensities (vs. VV) for the ten samples shown in the images of Figure 3, continued from previous page. 

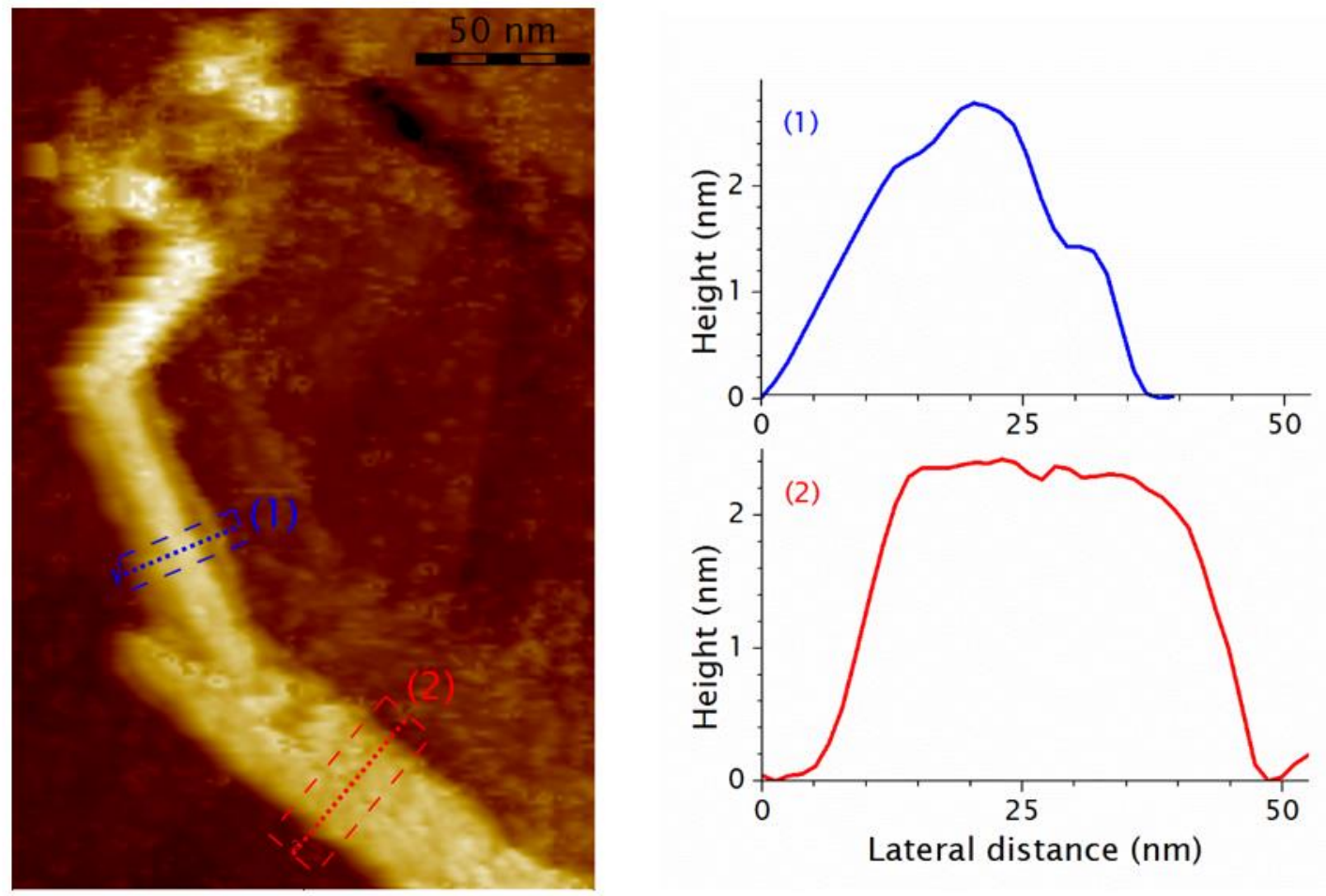

Figure S4. Left: Constant current $(+2.0 \mathrm{~V}, 10 \mathrm{pA})$ STM image of an aggregate deposited on Au(111) from $5 \mu \mathrm{M}$ TSPP in $0.75 \mathrm{M} \mathrm{LiCl}$ (aq). Right: Height profiles averaged over the correspondingly numbered/colored areas indicated in the image. 


\section{MATHEMATICAL DETAILS}

\section{A. Transition polarizability elements, depolarization ratios, and energy level structure}

The transition polarizability is a second rank tensor, and so has three rotational invariants:

$$
\begin{gathered}
\Sigma^{0}=\frac{1}{3}\left|\alpha_{x x}+\alpha_{y y}+\alpha_{z z}\right|^{2} \\
\Sigma^{1}=\frac{1}{2}\left[\left|\alpha_{x y}-\alpha_{y x}\right|^{2}+\left|\alpha_{y z}-\alpha_{z y}\right|^{2}+\left|\alpha_{z x}-\alpha_{x z}\right|^{2}\right] \\
\Sigma^{2}=\frac{1}{2}\left[\left|\alpha_{x y}+\alpha_{y x}\right|^{2}+\left|\alpha_{y z}+\alpha_{z y}\right|^{2}+\left|\alpha_{z x}+\alpha_{x z}\right|^{2}\right] \\
+\frac{1}{3}\left[\left|\alpha_{x x}-\alpha_{y y}\right|^{2}+\left|\alpha_{y y}-\alpha_{z z}\right|^{2}+\left|\alpha_{z z}-\alpha_{x x}\right|^{2}\right] .
\end{gathered}
$$

The depolarization ratio is written in terms of these quantities as

$$
\rho=\frac{5 \Sigma^{1}+3 \Sigma^{2}}{10 \Sigma^{0}+4 \Sigma^{2}}
$$

For a totally symmetric mode resonant with a transition to a nondegenerate electronic (excitonic) level, the transition polarizability has one nonzero, diagonal element. Defining the value of this element as $a$, equations 1 lead to $\Sigma^{2}=(1 / 3)|a|^{2}=2 \Sigma^{0}$ and $\Sigma^{1}=0$. Substituting in equation 2 gives $\rho=1 / 3$. For a totally symmetric mode resonant with a transition to a doubly degenerate electronic level, there are two nonzero transition polarizability elements, leading to $\Sigma^{0}(4 / 3)|a|^{2}=2 \Sigma^{2}$ and $\rho=1 / 8$. For nontotally symmetric modes resonant with only one electronic transition of any degeneracy the transition polarizability is symmetric, and its trace vanishes, so $\Sigma^{1}=\Sigma^{0}=0$, leaving only $\Sigma^{2}$ nonzero, which gives $\rho=3 / 4$.

When resonance with multiple electronic states occurs, interference between terms involving resonance with different electronic states contribute to the value of each tensor element, and no general statements can be made about depolarization ratio values. Transitions to a single electronic level permit the electronic transition dipole moment to be factored out of the sum over vibrational resonances (which is the same for all transition polarizability elements, and so can be cancelled in equation A2). This is not possible for resonance with multiple electronic transitions, which results in frequency dependence that differs among transition polarizability elements, and thus depolarization ratio dispersion (variation with excitation frequency). Differences in $\rho$ values and patterns of dispersion among samples indicate differences in underlying energy level structure. 


\section{B. Estimating RR depolarization ratios from oriented sample spectra}

The transition polarizability tensor components are complex quantities, i.e.,

$$
\alpha_{\rho \sigma}=a_{\rho \sigma}+i b_{\rho \sigma},
$$

whereas the square root of an integrated spectral intensity is a real quantity (modulus) related to the real and imaginary parts of the tensor element by

$$
\bar{\alpha}_{\rho \sigma} \equiv\left|\alpha_{\rho \sigma}\right|=\left(a_{\rho \sigma}^{2}+b_{\rho \sigma}^{2}\right)^{1 / 2} \text {. }
$$

By the triangle inequalities

$$
\begin{gathered}
\left|\alpha_{\rho \sigma}+\alpha_{\sigma \rho}\right| \leq\left|\alpha_{\rho \sigma}\right|+\left|\alpha_{\rho \sigma}\right|=\left|\bar{\alpha}_{\rho \sigma}+\bar{\alpha}_{\sigma \rho}\right| \\
\left|\alpha_{\rho \sigma}+\alpha_{\sigma \rho}\right|^{2} \leq\left|\bar{\alpha}_{\rho \sigma}+\bar{\alpha}_{\sigma \rho}\right|^{2}
\end{gathered}
$$

and

$$
\begin{gathered}
\left|\alpha_{\rho \sigma}-\alpha_{\sigma \rho}\right| \geq|| \alpha_{\rho \sigma}|-| \alpha_{\rho \sigma}||=\left|\bar{\alpha}_{\rho \sigma}-\bar{\alpha}_{\sigma \rho}\right| \\
\left|\alpha_{\rho \sigma}-\alpha_{\sigma \rho}\right|^{2} \geq\left|\bar{\alpha}_{\rho \sigma}-\bar{\alpha}_{\sigma \rho}\right|^{2},
\end{gathered}
$$

we can write the quantity estimated from the square roots of intensities as

$$
\bar{\rho}=\frac{5 \bar{\Sigma}^{1}+3 \bar{\Sigma}^{2}}{10 \bar{\Sigma}^{0}+4 \bar{\Sigma}^{2}} .
$$

Here, $\bar{\Sigma}^{1}$ is calculated from differences of terms $\bar{\alpha}_{\rho \sigma}$ and $\bar{\Sigma}^{0}$ from sums of such terms. The right hand side of inequalities $4 \mathrm{~b}$ and $5 \mathrm{~b}$ then imply that $\bar{\Sigma}^{1} \leq \Sigma^{1}$ and $\bar{\Sigma}^{0} \geq \Sigma^{0}$, making the numerator of the intensity-derived quantity smaller, and the denominator larger than would be calculated from the actual complex tensor components. Therefore we would expect $\bar{\rho} \leq \rho$ - estimates of depolarization ratios from RR intensities of fixed-orientation samples will underestimate the actual depolarization ratio.

However, $\Sigma^{2}$ has part that depends on sums of off-diagonal tensor elements and part that depends on differences of diagonal tensor elements. For apparently narrower structures (likely individual nanotubes) the diagonal element differences tend to be smaller than the off-diagonal element sums, whereas for the broader (likely bundled) structures, the sums of off-diagonal elements are greater than the differences of diagonal elements. This would should lead to a further underestimate of $\rho$ in the former case but a more uncertain situation in the latter (could be either an overestimate or underestimate). This means that comparisons of solution phase $\rho$ values among samples based on individual component intensties will involve more significant underestimates for the non-bundled specimens, but more uncertainty for bundled specimens. 
